\title{
Simulations numériques des tubes avec contraction brusque sur OpenFoam
}

\section{Numerical Simulations of Tubes with Abrupt Contraction on OpenFoam}

\author{
Tarik Chakkour ${ }^{1}$ \\ ${ }^{1}$ Université Bretagne Loire, Vannes, France, tarik.chakkour@univ-ubs.fr
}

RÉSUMÉ. La simulation du système moteur est un enjeu important dans l'industrie automobile car elle permet d'accélérer le processus de conception, et de limiter le recours aux prototypes des tests physiques. La plateforme AMESim permet d'accéder à des librairies de modèles permettant de simuler un moteur ou un véhicule. Les approches de modélisation (0D/1D) ne permettent pas cependant d'égaler les niveaux de précision des codes 3D. D'où l'étude sur OpenFoam (un logiciel de simulation CFD 3D) afin de ramener le problème en 1D, et d'effectuer un couplage 1D/3D en ouvrant de nouvelles perspectives de temps de calcul, et de qualité de prédiction.

ABSTRACT. The simulation engine system is an important issue in the automotive industry because of speeding up the design process, and limit the use of physical prototypes testing. The platform AMESim allows to access to libraries models for simulating engine or vehicle. Modeling approaches (OD/1D) do not however allow to match the 3D code accuracy levels. From this the study is on OpenFoam (simulation software CFD 3D) in order to reduce the problem in 1D with opening new perspectives of computing time, and prediction quality.

MOTS-CLÉS. CFD, orifices 3D, simulation numériques, OpenFoam, équations d'Euler

KEYWORDS. CFD, orifices 3D, numerical simulations, OpenFoam, Euler equations

\section{Introduction}

Le développement de la dynamique des fluides computationnelle (CFD) des méthodes à usage industriel est principalement dictée par les exigences d'effectuer des simulations sur un modèle à géométrie 3D, et de comportement physique complexes. La méthode des volumes finis est généralement préférée pour CFD industriels car elle est relativement peu coûteuse et se prête bien à la résolution des équations associées aux flux complexes. Habituellement, les variables comme la vitesse, et la pression sont colocalisées, c'est-à-dire qu'elles sont définies au même ensemble de mailles discrets (les centres des cellules).

La présence de discontinuités, telles que les chocs et les surfaces de contact, à haute vitesse compressible des flux nécessite des schémas numériques qui peuvent capturer ces caractéristiques tout en évitant les fausses oscillations. Notamment des méthodes numériques efficaces générant des solutions exactes non oscillatoire. Les méthodes qui impliquent généralement un solveur de Riemann ou une évaluation de la jacobienne les rend difficile à mettre en oeuvre dans un domaine polyédrique par exemple. Nous proposons des schémas numériques développés par Nessyahu et Tadmor (Haim 1990) comme une généralisation de deuxième ordre du schéma de Lax-Friedrichs. La méthode a été développée pour les systèmes multidimensionnels, et des maillages non structurés, principalement à partir de mailles de triangles, de tétraèdre, de quadrilatère et d'hexaèdre.

L'objectif de cet article est le développement, et l'étude des tubes avec des contractions brusques sur OpenFoam (Openfoam, 2016), un logiciel de simulation CFD 3D open source. Comme les approches de modélisation sur AMESim CFD 1D ne permettent pas cependant d'égaler les niveaux de précision 
des codes 3D. Pour cela, nous allons effectuer une étude complète sur des orifices en 3D. Ces orifices sont des tubes qui constituent des conduits d'échappement d'un moteur. Bien que la librairie AMESim permet d'observer des résultats assez fiables en terme de résolution des ondes et des chocs, elle ne peut cependant pas permettre de simuler les écoulements des gaz fortement 3D dans les systèmes plus compliqués comme des collecteurs d'échappement. Or, pour les constructeurs du moteur, il est très important de prendre en compte l'écoulement en 3D des gaz à l'intérieur des collecteurs pour prédire un meilleur rendement. D'où la nécessité de construire des modèles en utilisant un logiciel de CFD 3D et de ramener le problème en $1 \mathrm{D}$.

Il existe plusieurs études (Satish G, 2013, Guo B, 2001) des modèles de contraction brusque. Le document (Satish G, 2013) décrit le comportement d'écoulement d'un fluide en 2D dans un tube avec contraction brusque sur le logiciel Fluent CFD. Les résultats numériques générés ont été validés en comparaison aux données expérimentales. Dans l'article (Guo B, 2001), on étude un écoulement turbulent dans un tuyau suivi d'une contraction brusque. L'approche RANS transitoire et le modèle k- $\epsilon$ sont mises en oeuvre pour montrer l'instabilité de l'écoulement sur certaines zones.

\section{Modélisation des tubes avec contraction brusque}

\subsection{Les équations d'Euler}

Dans cette sous-section, nous déterminons les équations régissant la dynamique d'écoulement d'un fluide compressible non visqueux dans un canal plan tridimensionnel. L'objectif est d'obtenir une validation des résultats de la simulation numérique sur OpenFoam. Notre description s'inspire des équations d'Euler avec un schéma numérique aux volumes finis Kurganov et Tadmor (Alexander Kurganov and Eitan Tadmor, 2000, Alexander Kurganov and Guergana Petrova, 2000).

Les équations d'Euler de la dynamique des gaz permettent de décrire la conservation de la masse, de l'impulsion et de l'énergie sous forme locale en fonction de la masse volumique $\rho$, de la vitesse $\mathbf{u}$, de la pression $p$, de l'énergie interne $e$. L'énergie totale $E$ est la somme de l'énergie cinétique et interne. Dans le cas d'un gaz parfait polytropique, l'énergie $E$, la pression $p$ et l'énergie interne $e$ sont données par les relations suivantes :

$$
\left\{\begin{array}{l}
E=\frac{1}{2}\|\mathbf{u}\|^{2}+e \\
p=\rho R T \\
e=c_{v} \rho T
\end{array}\right.
$$

L'équation de la conservation de la masse :

$$
\frac{\partial \rho}{\partial t}+\nabla \cdot[\mathbf{u} \rho]=0
$$

L'équation de la conservation de la quatité du mouvement :

$$
\frac{\partial \rho \mathbf{u}}{\partial t}+\nabla \cdot[\mathbf{u}(\rho \mathbf{u})]+\nabla p=0
$$

L'équation de la conservation de l'énergie :

$$
\frac{\partial \rho E}{\partial t}+\nabla \cdot[\mathbf{u}(\rho E)]+\nabla \cdot[\mathbf{u} p]=0
$$


Nous pouvons considérer généralement une seule équation hyperbolique permettant la conservation des quantités physiques qui s'appellent des équations d'Euler :

$$
\frac{\partial w}{\partial t}+\nabla \cdot[F(w)]=0
$$

où $w$ est le vecteur des quantités conservées des variables, et $F$ est le tenseur de flux numérique, qui sont définis par :

$$
w=\left(\begin{array}{c}
\rho \\
\rho \mathbf{u} \\
\rho E
\end{array}\right), \quad F(w)=\left(\begin{array}{c}
\mathbf{u} \rho \\
\mathbf{u}(\rho \mathbf{u})+p I \\
\mathbf{u}(\rho E+p)
\end{array}\right) .
$$

La forme intégrale standard de l'équation d'Euler dans un volume de contrôle $V_{P}$ de centre $P$ est décrite sous la forme suivante :

$$
\int_{t}^{t+\Delta t}\left[\frac{d}{d t} \int_{V_{P}} w \mathrm{~d} V+\int_{V_{P}} \nabla \cdot[F(w)] \mathrm{d} V\right] \mathrm{d} t=0 .
$$

\subsection{Stabilité du solveur}

Plusieurs solveurs concernant des écoulements compressibles sont disponibles dans la distribution du logiciel OpenFoam, chacun est adapté à un type spécifique d'écoulement compressible. Le solveur rhopSonicFoam s'utilise comme base d'un solveur compressible microflux, comme il l'a été partiellement réaménagé d'analyse des flux dans les applications de l'aérodynamique hypersonique. Le solveur rhopSonicFoam est une application de pression basée sur la densité des écoulements laminaires compressibles. Il résout les équations régissant la continuité, la quantité du mouvement (posée en termes de masse de flux), et de l'énergie totale. Ces équations sont résolues dans une manière distincte, suivie d'une boucle de correction de pression (algorithme PISO décrit dans (Marie-Pierre Dainese, 1994).

Les deux solveurs rhoSonicFoam et rhopSonicFoam sont basés sur l'équation de pression pour les problèmes transsonores, y compris des régions de faible nombre de Match. SonicFoam est un solveur qui résout les équations de Navier-Stokes avec l'algorithme PISO. Si la viscosité est nulle, il résout les équations d'Euler d'un fluide non visqueux compressible. Le solveur SonicFoam calcule simplement l'énergie interne $e$ (l'énergie thermique). L'énergie cinétique est ignorée et n'est pas calculée.

Nous voulons maintenant localiser le solveur le plus stable afin de modéliser un écoulement compressible non visqueux avec les équations d'Euler. Pour cela, nous configurons avec des conditions aux limites de conduit bouché un problème de tube à choc. La Figure 1 présente l'évolution d'une onde de choc de la variable pression pour les trois solveurs, SonicFoam, rhoSonicFoam et rhopSonicFoam. Nous effectuons cette simulation pour le même pas de temps et d'espace. Comme la courbe de la pression du schéma SonicFoam est présentée en bleu, nous concluons qu'il est le solveur le plus stable. 


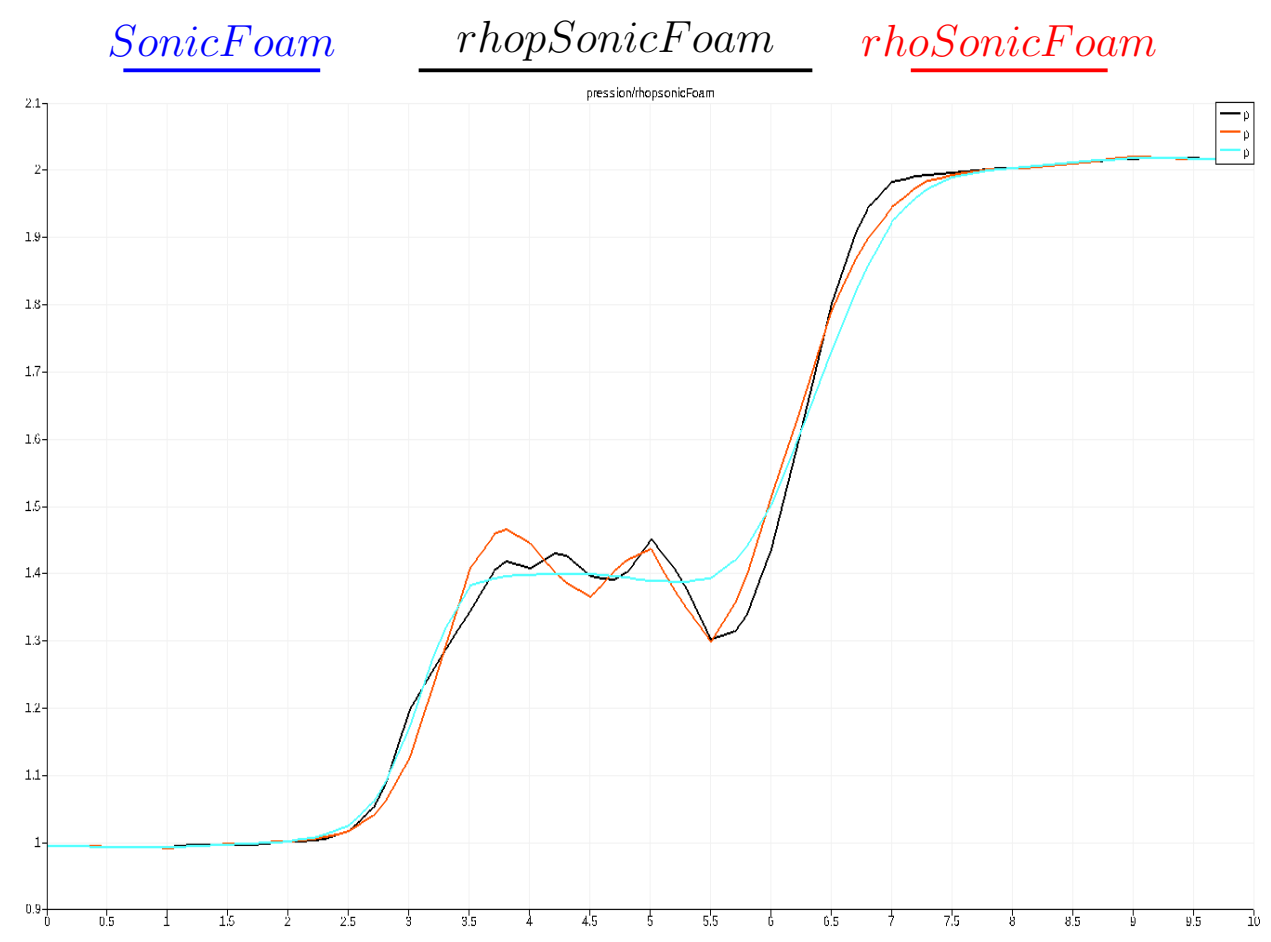

Figure 1. Comparaison des trois solveurs

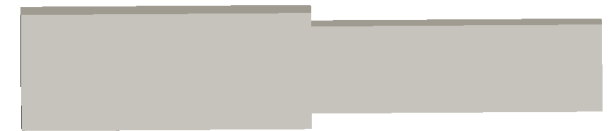

Figure 2. Domaine cartésien

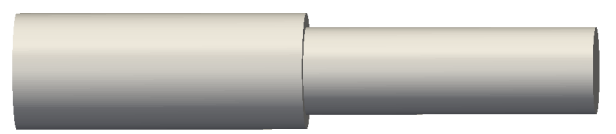

Figure 3. Domaine cylindrique

\subsection{La présentation du modèle}

Soit $S$ une section donnée. Nous définissons un modèle de contraction lorsque la variation de la section est négative $d S<0$, et nous définissons un modèle d'élargissement lorsque la variation de la section est positive $d S>0$. Notre domaine physique est un conduit horizontal de longueur $0.2 m$ subissant une contraction en $x=0.1 \mathrm{~m}$ dont la moitié gauche a un diamètre de $0.04 \mathrm{~m}$, et dont la moitié droite a un diamètre de $0.03 \mathrm{~m}$. Nous précisons que le quotient de carré de la moitié droite, et celle de la moitié gauche doit être supérieur à $0.5 \mathrm{~m}$.

Nous considérons deux domaines de calcul qui sont un domaine cartésien, et un domaine cylindrique pour savoir si la géométrie du domaine de calcul influence la nature de notre écoulement moyen. Ensuite, nous réalisons deux différents types du maillage (lâche et raffiné) sur ces deux domaines de calcul pour savoir si notre maillage est convergent par rapport à notre simulation numérique. Ce maillage est un 
maillage équidistribué suivant les trois directions de l'espace : $\Delta x=\Delta y=\Delta z$ avec des longueurs variées sur notre domaine de calcul.

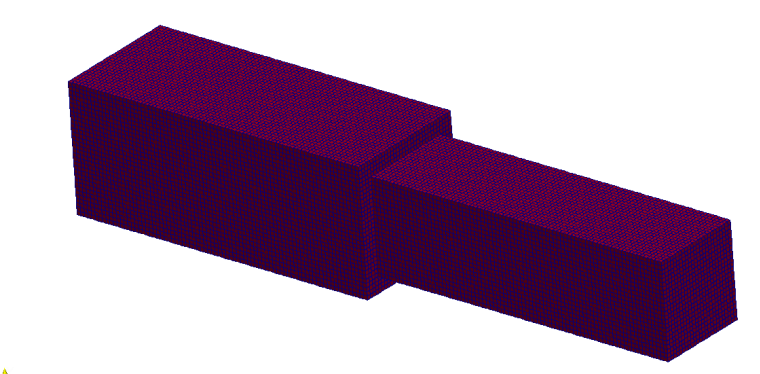

Figure 4. Maillage cartésien lâche

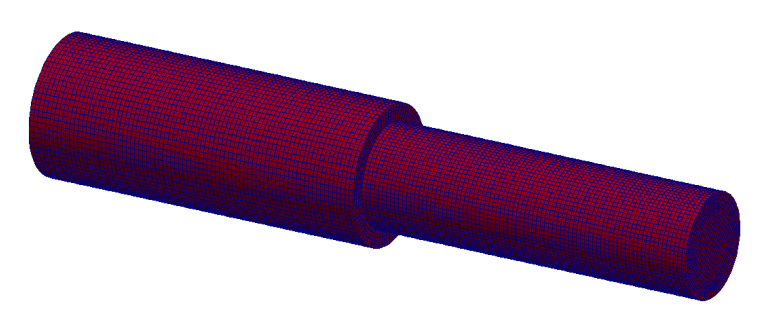

Figure 6. Maillage cylindrique lâche

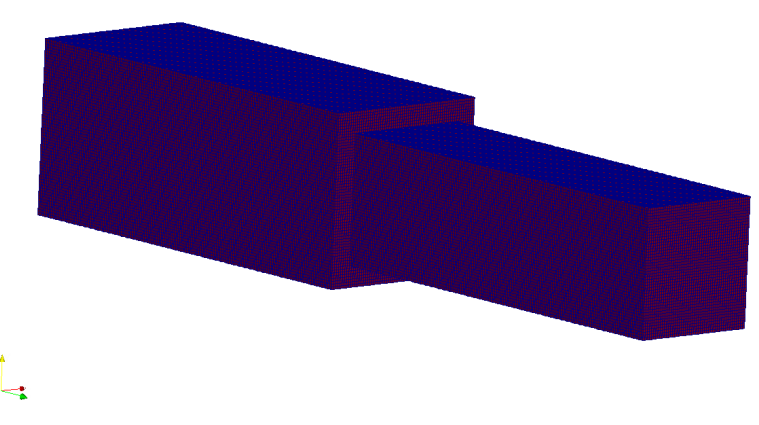

Figure 5. Maillage cartésien raffiné

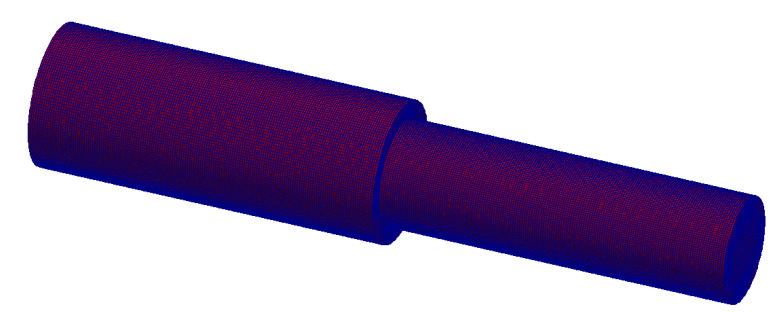

Figure 7. Maillage cylindrique raffiné

Le domaine de calcul est subdivisé en plusieurs volumes de contrôles de forme, et de taille quelconques. Ces cellules doivent couvrir l'ensemble du domaine de calcul pour fournir une bonne approximation de la géométrie de l'écoulement. Pour un maillage lâche, nous discrétisons la petite section avec un nombre de mailles $n_{x} \times n_{y} \times n_{z}$ et la grande section avec un nombre de mailles $m_{x} \times m_{y} \times m_{z}$, dans le repère $\left(\overrightarrow{e_{x}}, \overrightarrow{e_{y}}, \overrightarrow{e_{z}}\right)$. Nous notons $\Delta t$ le pas de temps de discrétisation. Plus formellement :

$$
\left\{\begin{array}{l}
\text { Maillage lâche } \\
\Delta x=\Delta y=\Delta z=0.00125 m \\
n_{x}=12, n_{y}=12, n_{z}=80 \\
m_{x}=16, m_{y}=16, m_{z}=80 \\
\Delta t=2.68 \times 10^{-6}
\end{array}\right.
$$

Pour un maillage raffiné, nous discrétisons la petite section avec un nombre de mailles $n_{x} \times n_{y} \times n_{z}$, et la grande section avec un nombre de mailles $m_{x} \times m_{y} \times m_{z}$ dans le repère $\left(\overrightarrow{e_{x}}, \overrightarrow{e_{y}}, \overrightarrow{e_{z}}\right)$ : 


$$
\left\{\begin{array}{l}
\text { Maillage raffiné } \\
\Delta x=\Delta y=\Delta z=0.000625 m \\
n_{x}=24, n_{y}=24, n_{z}=160 \\
m_{x}=32, m_{y}=32, m_{z}=160 \\
\Delta t=1.34 \times 10^{-6}
\end{array}\right.
$$

\subsection{Les conditions aux limites et initiales}

Nous voulons que l'écoulement soit stationnaire où le fluide circule de gauche à droite avec une vitesse initiale nulle. Pour cela, nous imposons des conditions aux limites symmetryPlane sur la surface latérale afin que l'écoulement soit unidimensionnel. Nous distinguons deux types de conditions aux limites et initiales :

- Nous imposons une différence de température $\Delta T$ à $57 \mathrm{~K}$ avec un vecteur gradient de pression nul, et une vitesse d'entrée fixée à $50 \mathrm{~m} / \mathrm{s}$, et un vecteur gradient de pression d'entrée nul. Nous imposons sur la sortie un vecteur gradient de vitesse, et de température nul. Nous présentons ces conditions aux limites et initiales dans la Figure 8 :

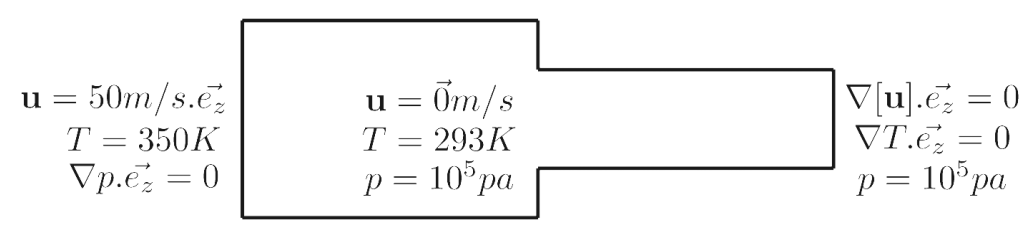

Figure 8. Conditions aux limites et initiales I

- Nous définissons dans ce cas des conditions initiales stationnaires. Pour cela, nous imposons une différence de température $\Delta T$ de $300 K$ avec une pression de sortie constante fixée à $p=10^{5} \mathrm{pa}$, et une vitesse d'entée relativement très grande. Nous présentons ces conditions aux limites et initiales dans la Figure 9 :

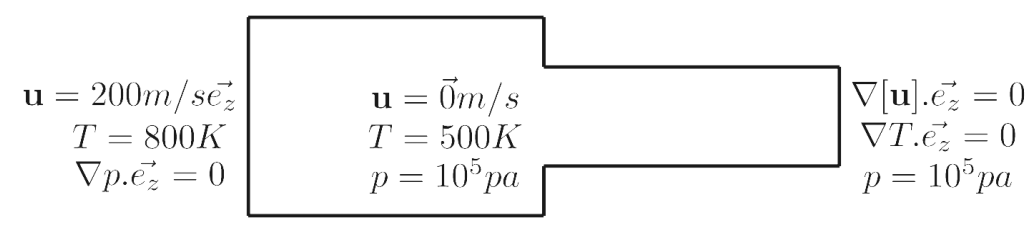

Figure 9. Conditions aux limites et initiales II

Soit $S_{i}$ la surface de chaque cellule $C$ d'une section qui est orthogonale à la direction $\overrightarrow{e_{z}}$ de l'écoulement de notre tube. Si $v$ est une variable présentant une quantité physique, nous définissons la variable moyenne $\bar{v}$ par la moyenne surfacique de $v$ sur chaque cellule $C$ sous la forme suivante :

$$
\bar{v}=\frac{\sum_{i \in C} v_{z, i} S_{i}}{\sum_{i \in C} S_{i}} .
$$


Nous utilisons la relation [8] pour définir la vitesse moyenne $\overline{\mathbf{u}}_{z}$, la densité moyenne $\bar{\rho}_{z}$ et la pression moyenne $\bar{p}_{z}$ respectivement à partir de la vitesse $\mathbf{u}_{z}$, de la densité $\rho_{z}$ et de la pression $p_{z}$. Nous rappelons que le débit $Q$ est défini par l'intégrale de la densité fois la vitesse dans la direction de l'écoulement $\vec{e}_{z}$ sur l'élément de surface $d S$

$$
Q=\int_{S} \rho \mathbf{u}_{z}
$$

À partir de la définition [9] du débit moyen, nous en distinguons deux expressions $D$ et $d$. Le premier débit $D$ est pris en considération des fluctuations et est défini à partir de la moyenne de la vitesse fois la densité $\overline{\rho_{z} \mathbf{u}_{z}}$ par :

$$
D=\overline{\rho_{z} \mathbf{u}_{z}} \times S .
$$

Nous allons maintenant justifier l'expression du débit moyen $D$ définie dans la relation [10]. Le débit moyen peut se définir sous la forme :

$$
\bar{Q}=\int_{S} \overline{\rho \mathbf{u}_{z}} .
$$

Si nous intégrons sur une section constante $S$, nous obtenons l'expression [10] du débit moyen $D$. Nous allons maintenant justifier l'expression du deuxième débit $d$ étant défini par le produit de la densité moyenne $\bar{\rho}_{z}$ par la vitesse moyenne $\overline{\mathbf{u}}_{z}$ et la surface $S$ :

$$
d=\bar{\rho}_{z} \overline{\mathbf{u}}_{z} \times S .
$$

Nous rappelons que la conservation de la masse impose que le débit d'un fluide dans un conduit est constant. Si nous décomposons la vitesse instantannée $\mathbf{u}_{z}$ sous forme d'une somme de la vitesse moyenne $\overline{\mathbf{u}}_{z}$, et de la vitesse fluctuante $\underline{\mathbf{u}_{z}}$ telle que la moyenne d'une vitesse fluctuante est nulle, i.e : $\mathbf{u}_{z}=\overline{\mathbf{u}}_{z}+\underline{\mathbf{u}_{z}}$. Nous obtenons :

$$
\bar{Q}=\int_{S}(\bar{\rho}+\underline{\rho})\left(\overline{\mathbf{u}}_{z}+\underline{\mathbf{u}_{z}}\right) .
$$

Ensuite, nous simplifions l'expression du débit moyen $\bar{Q}$ définie dans la relation [13] pour obtenir la relation suivante :

$$
\bar{Q}=\int_{S} \bar{\rho} \overline{\mathbf{u}}_{z}+\int_{S}\left(\underline{\rho} \cdot \overline{\mathbf{u}}_{z}+\underline{\rho} \cdot \underline{\mathbf{u}_{z}}+\bar{\rho} \cdot \underline{\mathbf{u}_{z}}\right) .
$$


Le premier intègrale se simplifie puisque les valeurs moyennes sont constantes sur la section $S$. La deuxième partie de l'intègrale est nulle par passaqge à la moyenne d'une quantitée fluctuante. En effet, comme nous avons :

$$
\int_{S} \frac{\left(\underline{\rho} \cdot \overline{\mathbf{u}}_{z}+\underline{\rho} \cdot \underline{\mathbf{u}_{z}}+\bar{\rho} \cdot \underline{\mathbf{u}}_{z}\right)}{=0,}
$$

nous obtenons l'expression [12] du débit moyen $d$ sur une section constante. Similairement, nous distinguons deux expressions de l'enthalpie moyenne $H$ et $h$. La première enthalpie $H$ est prise en considération des fluctuations et est définie à partir de la moyenne de la quantité $E+\frac{p}{\rho}$ :

$$
H=\overline{E+\frac{p}{\rho}} .
$$

Cette expression de l'enthalpie moyenne $H$ peut se simplifie sous la forme suivante :

$$
H=\bar{E}+\frac{\bar{p}}{\rho} .
$$

La deuxième enthalpie $h$ est définie par la somme de l'énergie totale moyenne $\bar{E}$ et du quotient de la pression moyenne $\bar{p}$ et la densité moyenne $\bar{\rho}$

$$
h=\bar{E}+\frac{\bar{p}}{\bar{\rho}} .
$$

\section{Résultats numériques}

Nous voulons dans cette section évaluer l'enthalpie et le débit moyen au cours du temps et d'espace à travers des différentes positions qui sont proches de changement de section. La conservation d'enthalpie $h($ ou $H$ ) est équivalente à la conservation de la quantité de mouvement. Ainsi, la conservation de l'énergie totale est équivalente à la conservation d'entropie $s($ ou $S)$.

Nous considèrons dans la Figure 10 des positions amonts et avales de l'écoulement numérotées 1, 2 et 3. La position avale représente la position où le fluide s'écoule vers la direction de l'écoulement. Tandis que la position amont est définie par la position du sens opposé de l'écoulement. Les positions amonts 1,2 et 3 s'éloignent du changement de section respectivement par rapport à $3 \Delta z, 2 \Delta z$ et $\Delta z$. Nous utilisons la même définition concernant les position avales 1,2 et 3 .

Nous présentons dans la Figure 11 la section de chaque position amont at avale afin d'effectuer la moyenne de chacune des variables $\rho, \mathbf{u}_{z}$ et $\rho \mathbf{u}_{z}$. Cela permet de fournir le débit moyen $D$ calculé à partir de la moyenne de $\overline{\rho \mathbf{u}_{z}}$. Ainsi, le débit moyen $d$ calculé autrement en multipliant la densité moyenne sur la section $\bar{\rho}$, et la vitesse moyenne $\overline{\mathbf{u}}_{z}$ et la surface de la section. 


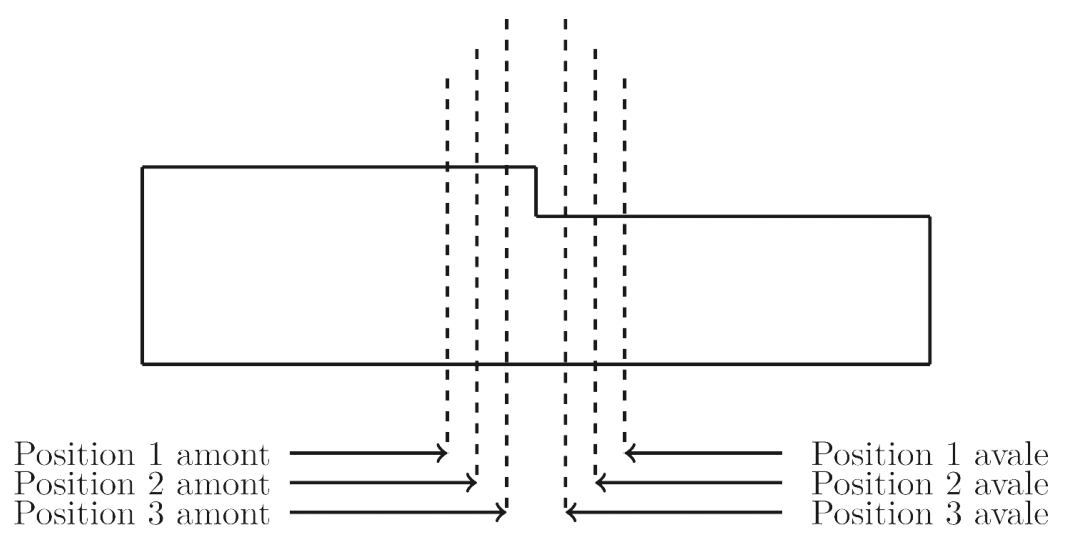

Figure 10. Positions amonts et avales

\subsection{Résultats avec des conditions aux limites et initiales I}

\subsubsection{La variation du débit moyen et de l'enthalpie moyenne}

Nous voulons dans cette sous-sous-section montrer comment le débit moyen et l'enthalpie moyenne varient au cours d'espace défini entre la position amont 1 , et la position avale 1 . Nous étudions la variation de ce débit moyen sur des différentes itérations temporelles. En effet, nous voulons montrer que le débit moyen, et que l'enthalpie moyenne sont conservées. Pour cela, nous traçons le débit moyen dans les Figures 12 et 13, et l'enthalpie moyenne dans les Figures 14 et 15 en fonction de cet espace défini. Nous remarquons que le débit massique est conservé uniquement entre deux sections du tube. Cette loi de conservation est définie par l'équation de continuité. Similairement, la conservation de l'enthalpie est équivalente à la conservation de la quantité de mouvement. Les résultats de la simulations affirment qu'il existe des instants où le débit moyen (respectivement l'enthalpie moyenne) n'est pas du tout conservé (respectivement conservée). Ces instants se coïncident avec le passage de l'onde au moment du changement de section.

Les simulations numériques définies dans les Figures 16, 17, 18, 19, 20, 21, 22, 23,24 s'effectuent par rapport à un schéma temporel d'Euler d'ordre 1. Nous choisissons le pas de temps pour un maillage

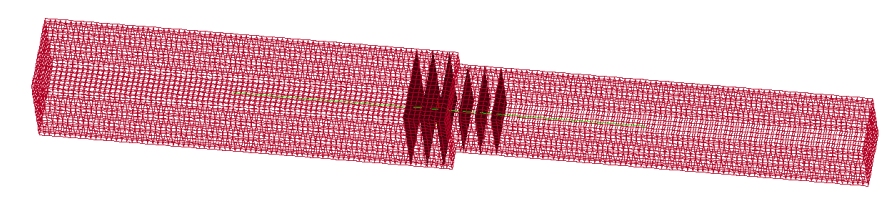

Figure 11. Différentes sections montrant les positions amonts et avales 1, 2, 3 


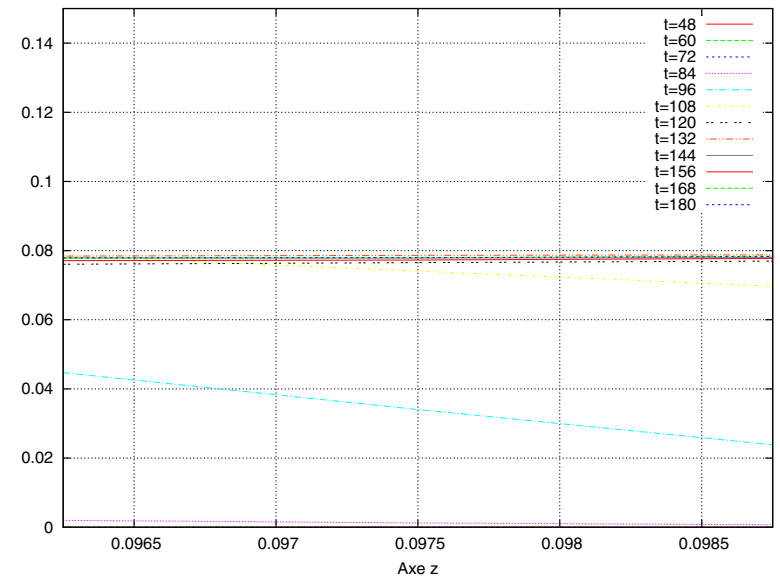

Figure 12. Débit moyen au cours de l'espace pendant un aller

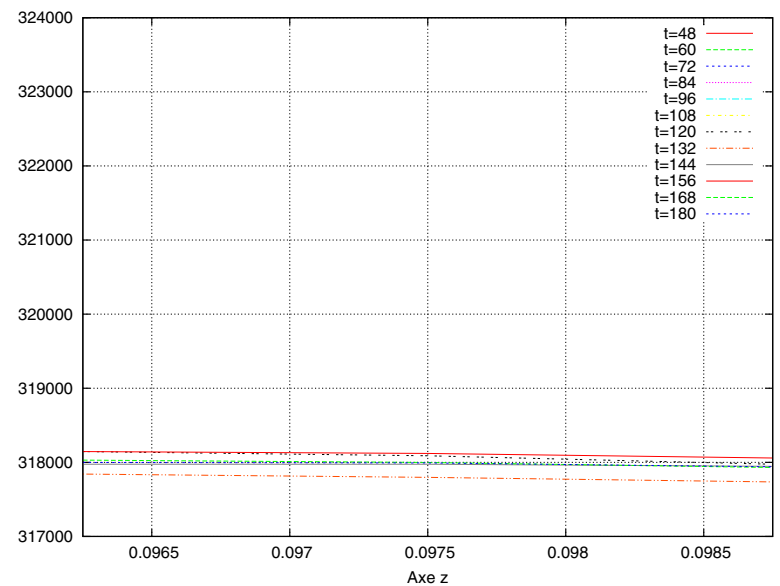

Figure 14. Enthalpie moyenne au cours de l'espace pendant un aller

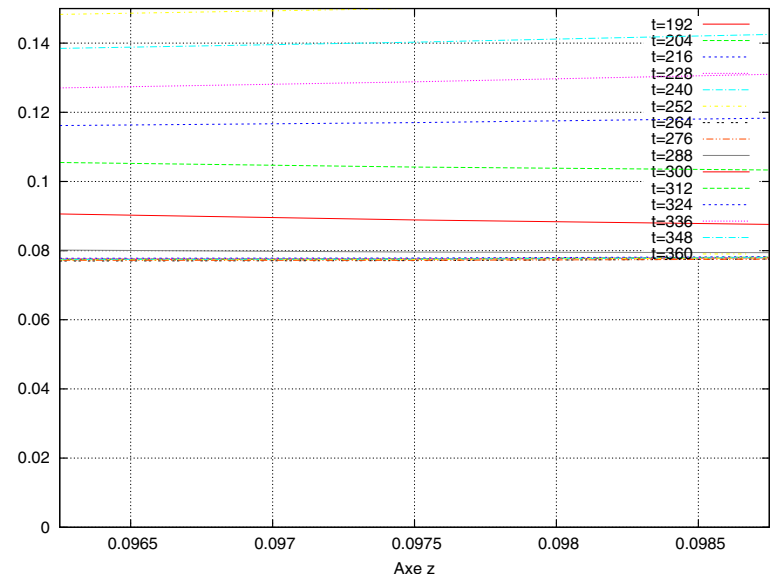

Figure 13. Débit moyen au cours de l'espace pendant un retour

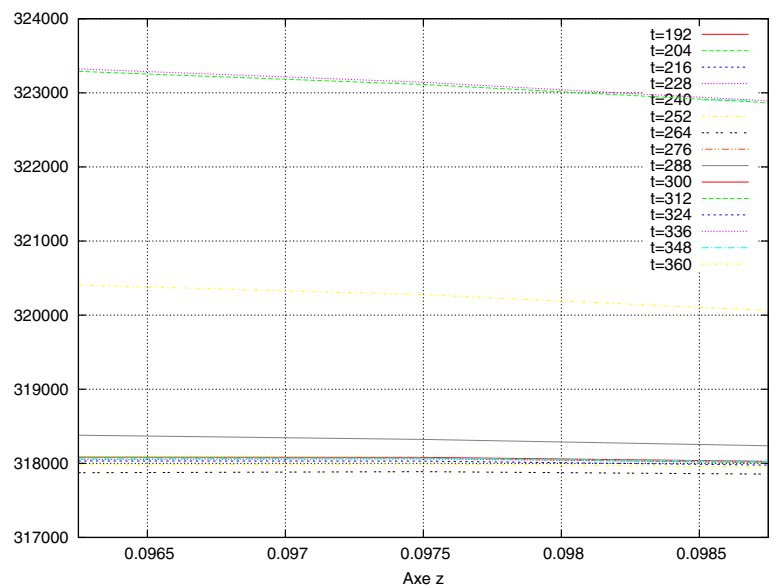

Figure 15. Enthalpie moyenne au cours de l'espace pendant un retour

lâche le double du maillage raffiné afin d'effectuer une simulation IsoCFL. Nous présentons ces résultats numériques du débit moyen au cours de chaque pas de temps $4 \times \Delta t$. Nous précisons que l'onde de retour dépense plus de temps par rapport à l'onde d'aller. Nous présentons dans ces Figures les deux débits moyens $D$ et $d$ définis respectivement dans les relations [10] et [12]. Une comparaison du débit moyen entre les deux Figures 21, 22 pour la poisition 1, et entre les deux Figures 23, 24 pour la poisition 2 montre que la géométrie du domaine de calcul n'influence pas la nature de notre écoulement moyen.

Concernant un maillage cartésien lâche, nous simulons la variation du débit moyen au cours du temps. Nous constatons pour des différentes positions 1,2,3 que le débit moyen est moins diphasé si nous nous approchons du changement de section, et il est plus diphasé si nous nous éloignons plus du changement de section pendant l'état stationnaire. Lorsque l'onde effectue un aller, il y a principalement deux régimes physiques qui s'installent. Un régime stationnaire dont le débit est nul, suivi d'un régime transitoire qui diphase les deux débits amont et aval. Lorsque l'onde se réfléchit, le régime devient stationnaire. Nous nous plaçons pour la suite dans un régime transitoire.

Nous choisissons un intervalle de simulation de longueur de pas temps $\Delta t$ afin d'estimer le diphasage entre le débit amont et aval. Nous prennons par défault un maillage lâche. Concernant un maillage cartésien raffiné, nous observons le même comportement pour le débit moyen que pour un maillage lâche. Il y 
a simplement une différence au 110 pas temps. L'onde n'a pas encore terminé son trajet pour l'aller. Le schéma reste quand même convergent au niveau du maillage par rapport à notre simulation numérique. Si nous nous approchons plus du changement de section, nous constatons aucune fluctuation du débit moyen. Nous concluons que notre approche est relativement convergente par rapport à notre maillage.

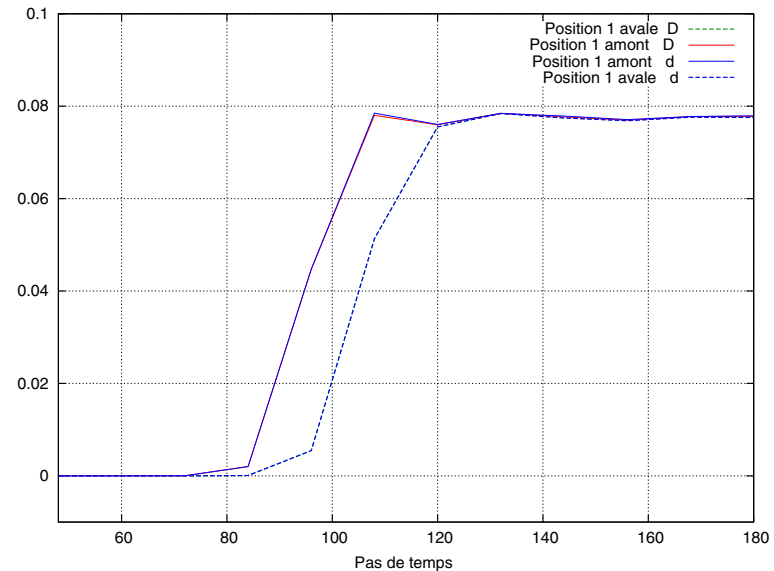

Figure 16. Débit moyen pour la poisition 1 pendant un aller

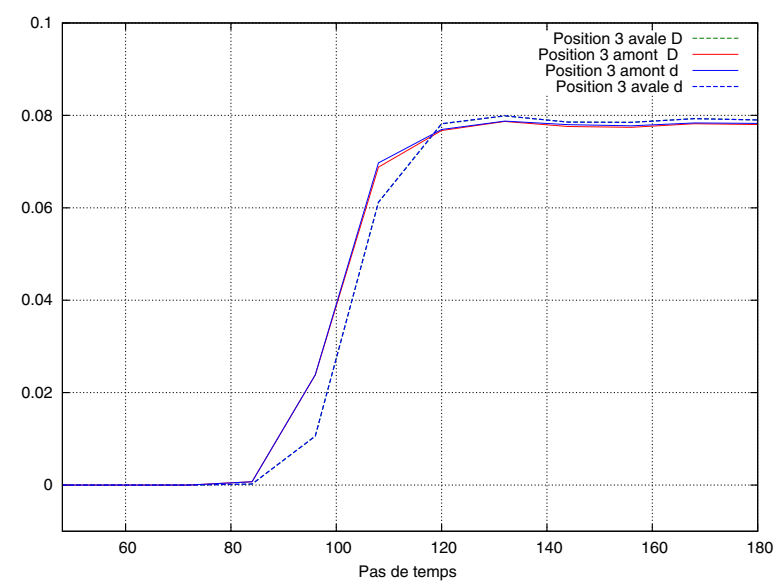

Figure 18. Débit moyen pour la poisition 3 pendant un aller

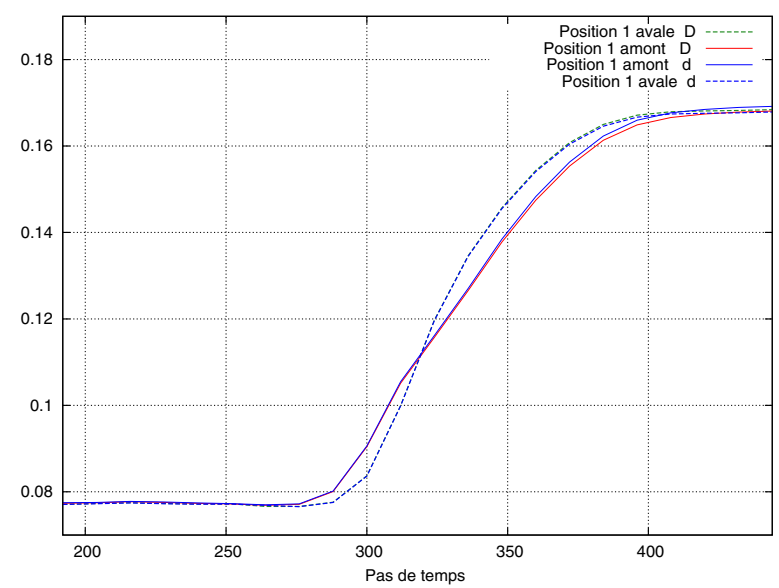

Figure 17. Débit moyen pour la position 1 pendant un retour

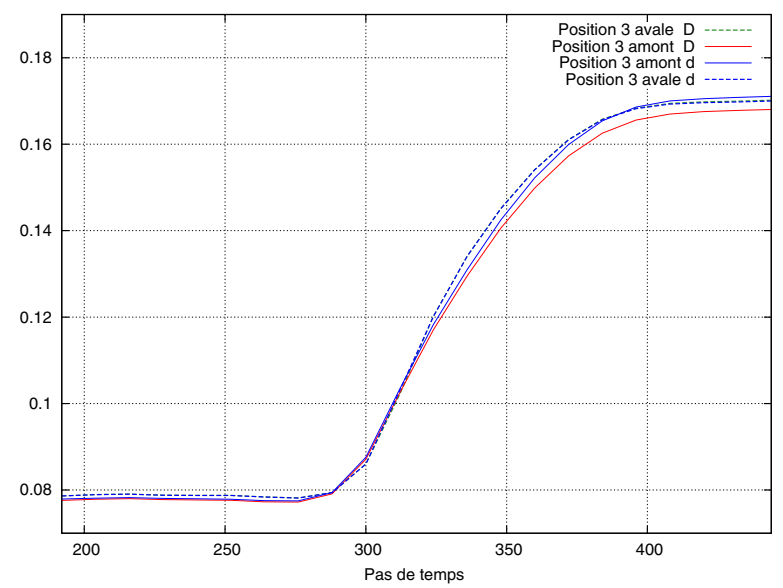

Figure 19. Débit moyen pour la position 3 pendant un retour

\subsubsection{La variation de la masse moyenne}

Nous voulons calculer la masse $m\left(V_{i}, t\right)$ comprimée dans chaque volume $V_{i}$ pour toute position $i$ amont et avale comprise entre 1 et 3 . Nous la calculons pour savoir si le diphasage trouvé sur le profil du débit est du à un problème physique ou à quelque chose d'autre. La masse $m\left(V_{i}, t\right)$ est définie respectivement sur chaque volume $V_{i}$ par:

$$
\forall i \in\{1,2,3\}, m\left(V_{i}, t\right)=\int_{V_{i}} \rho
$$




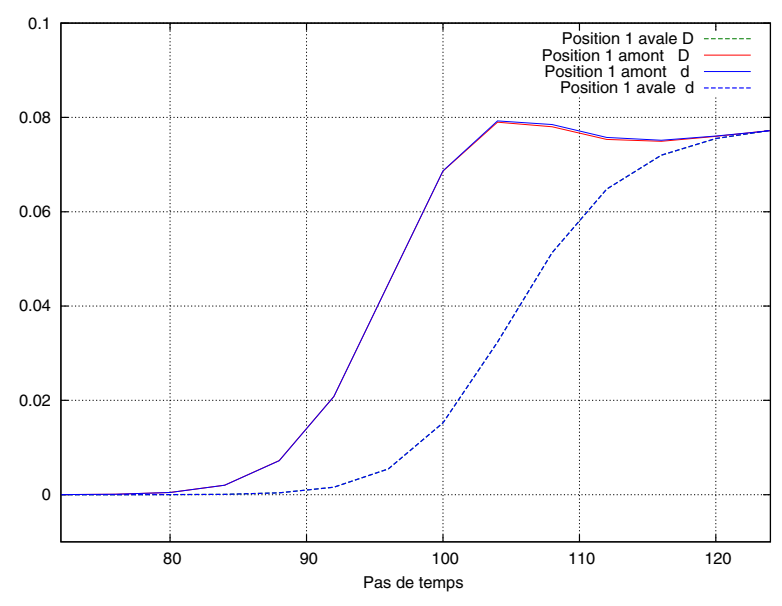

Figure 20. Débit moyen pour la poisition 1 pendant un aller

Nous calculons la masse moyenne $m\left(V_{i}, t\right)$ à l'aide de l'opérateur d'intégration. Nous présentons cette masse $m\left(V_{i}, t\right)$ au cours du temps dans la Figure 26. Cette simulation numérique montre que la masse $m\left(V_{1}, t\right)$ comprimée dans le volume $V_{1}$ est plus grande que les masses $m\left(V_{2}, t\right)$ et $m\left(V_{3}, t\right)$. Cette masse $m\left(V_{1}, t\right)$ est bien conservée pendant un régime établi. Cependant, elle n'est pas conservée au cours d'un régime instationnaire. Ce qui n'est pas cohérent en raison du diphasage du débit moyen pendant cette période.

\subsubsection{Les glyphes et les lignes de contour}

Nous voulons expliquer dans cette sous-sous-section ce qui se passe sur la partie du changement de section. Cette partie est à l'origine de l'état instationnaire du débit moyen avant que le régime soit établi. En effet, notre objectif est de déterminer la nature de l'écoulement proche de changement de section.

Le champ vectoriel de vitesse sur cette partie est caractérisé par son redirection pour passer à la deuxième section. Les vecteurs contournent cette zone afin d'éviter d'heurter le bord. Avec des conditions initiales stationnaires des équations d'Euler, les zones de circulation ne sont pas créées. Cependant, avec des conditions initiales instationnaires, elles sont créées. Concernant un maillage raffiné, nous obtenons un champ vectoriel distribué de manière aléatoire. Or, pour un maillage lâche, les vecteurs de vitesses sont totalement réguliers. En effet :

- La simulation numérique sur un maillage raffiné montre que l'écoulement est quasi 1D, et que le champ de vitesse suit une seule direction. Sauf sur la paroi où l'écoulement représente un détournement pour la vitesse suivant la direction de l'écoulement. Le champ de vitesse contournera la section du passage en gardant la même direction. Au centre du domaine, il y a un changement de sens de direction pour certains vecteurs de vitesse, et cela est dû à l'écoulement 2D.

- La direction de l'écoulement reste globalement régulière selon l'axe $\vec{e}_{z}$ pour un maillage lâche. Nous concluons clairement que l'écoulement pour un maillage lâche diffère du celui raffiné.

Les lignes de contour représentent les lignes que le fluide les contournent, les lignes horizontales montrent que c'est un écoulement 1D. Cependant, les lignes encerclées signifient que l'écoulement est en 3 dimensions d'espace. Pour le maillage raffiné, ça représente relativement une cercle dans la deuxième partie de l'écoulement. Ce qui montre qu'il y a un écoulement qui se développe dans cette 


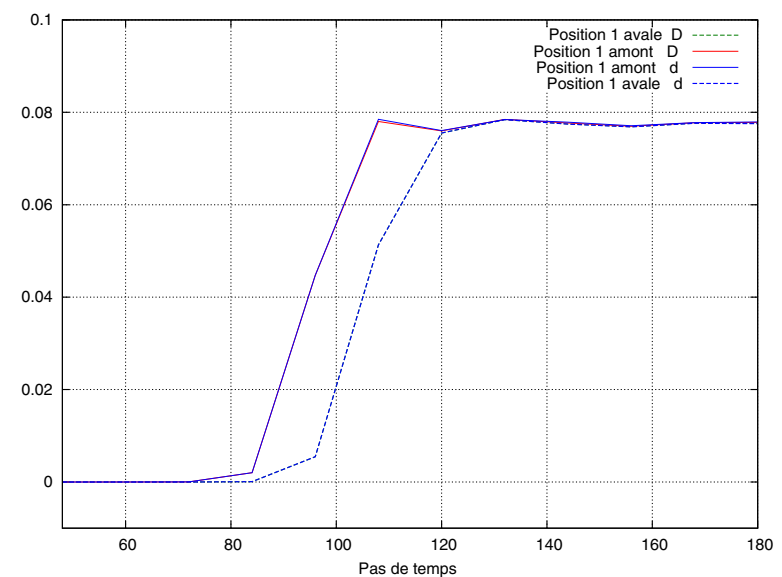

Figure 21. Débit moyen pour la poisition 1 pour un maillage lâche pendant un aller

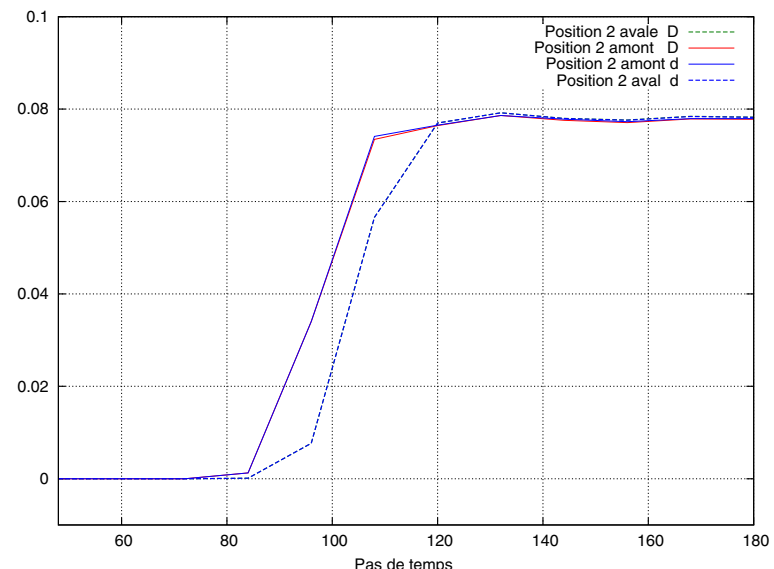

Figure 23. Débit moyen pour la poisition 2 pour un maillage lâche pendant un aller

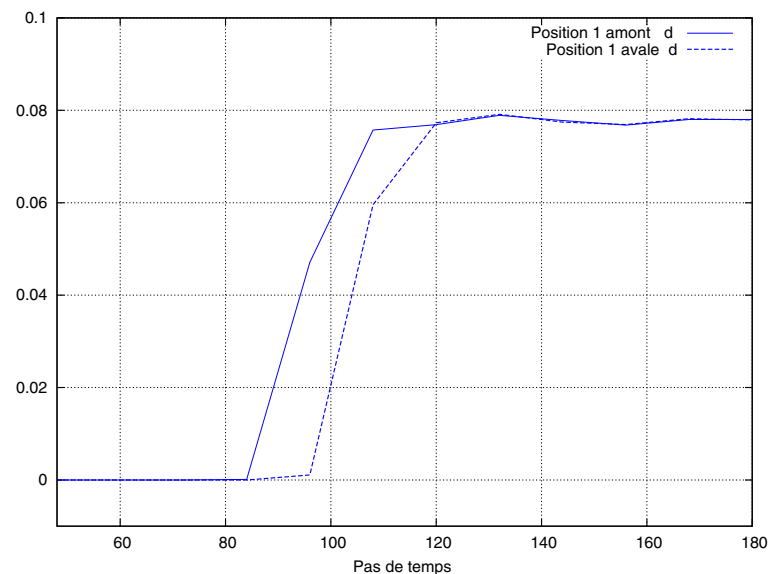

Figure 22. Débit moyen pour la position 1 pour un maillage raffiné pendant un aller

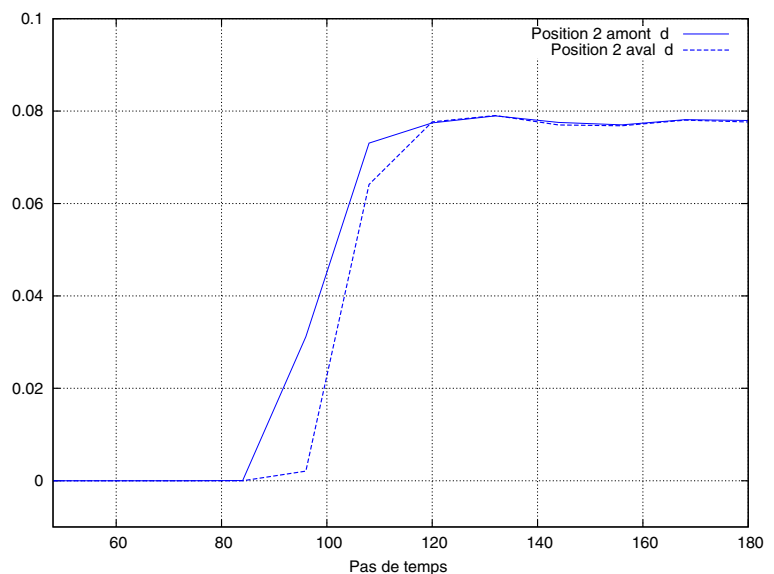

Figure 24. Débit moyen pour la position 2 pour un maillage raffiné pendant un aller
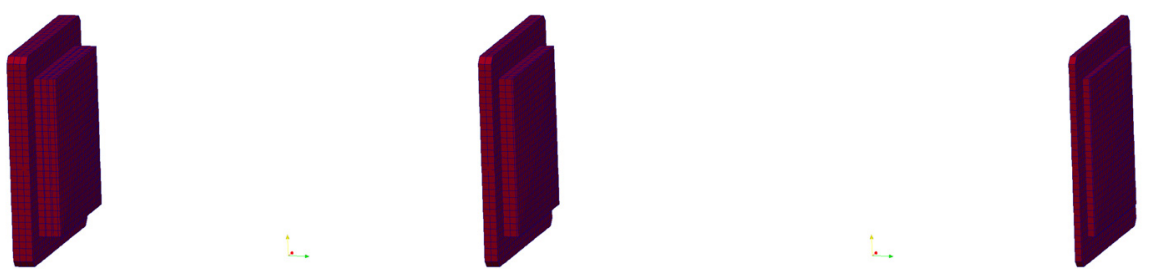

Figure 25. La figure présente trois diagrammes. Le diagramme du gauche présente le volume compris entre la position 1 amont et avale, le diagramme du milieu présente le volume compris entre la position 2 amont et avale, le diagramme du droit présente le volume compris entre la position 3 amont et avale

partie en comparaison avec le même écoulement pour un maillage lâche où il y a moins des lignes de contour.

\subsubsection{Les isosurfaces}

Les isosurfaces servent à déterminer la nature de l'écoulement en 3D. Comme nous en avons du mal à observer cela en 2D, nous effectuons ce type de simulation pour mieux les observer sur les lignes de contour et sur le profil de vitesse. Nous construirons des isosurfaces définies les Figures 31 et 32 pour 


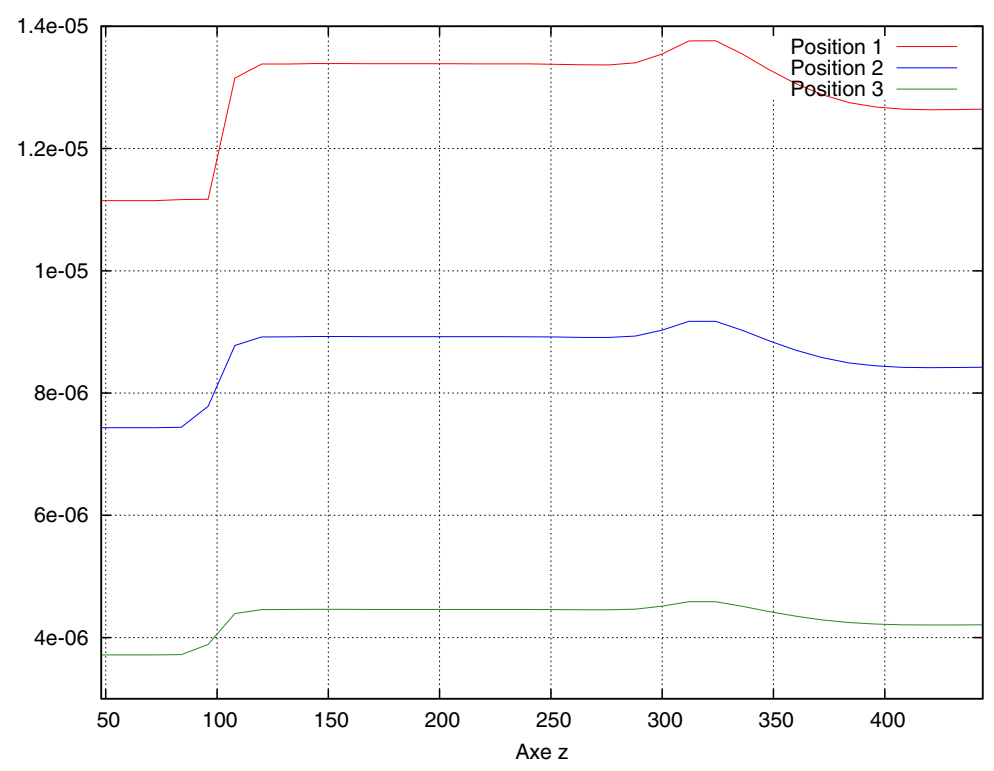

Figure 26. Variation de la masse $m\left(V_{i}, t\right)$ au cours du temps

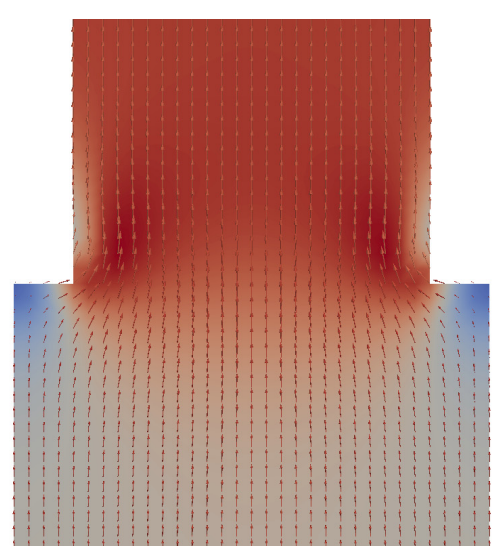

Figure 27. Champs vectoriel de vitesse pour un maillage lâche

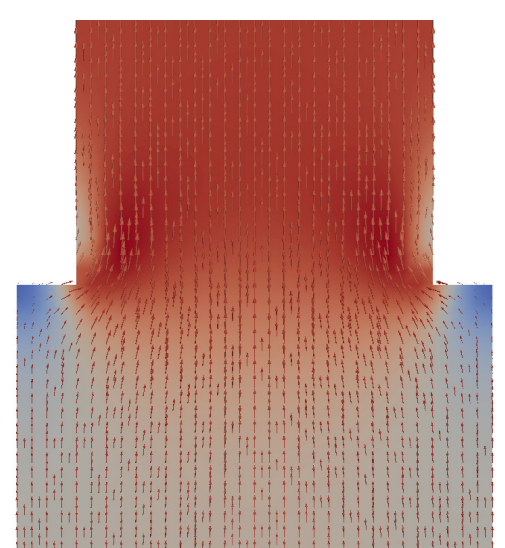

Figure 28. Champs vectoriel de vitesse pour un maillage raffiné

apporter une précision dans l'approximation de l'espace que le fluide traverse. Cet espace est découpé en plusieurs unités de volume.

Les isosurfaces en 3D associées à un maillage lâche montrent que l'écoulement est unidimensionnel en raison des plans parallèles distribués dans les deux différentes sections. Néanmoins, les contours en $3 \mathrm{D}$ pour un maillage raffiné affirment que nous nous plaçons dans un écoulement $2 \mathrm{D}$ parce que les plans se déforment, et finalement ne peuvent pas être parallèles.

\subsection{Résultats avec des conditions aux limites et initiales II}

\subsubsection{Le maillage du quart du domaine}

Le calcul sur le domaine entier prend considérablement du temps, et de place mémoire. Pour cela, nous considérons pour les conditions II que le domaine est le quart. Nous calculons dans la Figure 33 le temps 


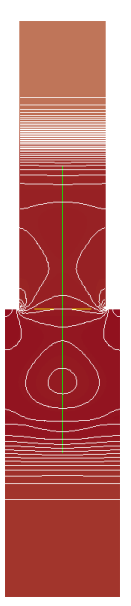

Figure 29. Lignes de contour pour un maillage lâche
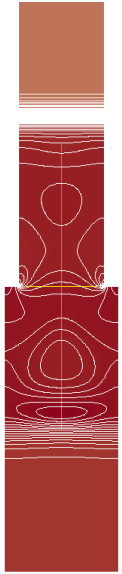

Figure 30. Lignes de contour pour un maillage raffiné

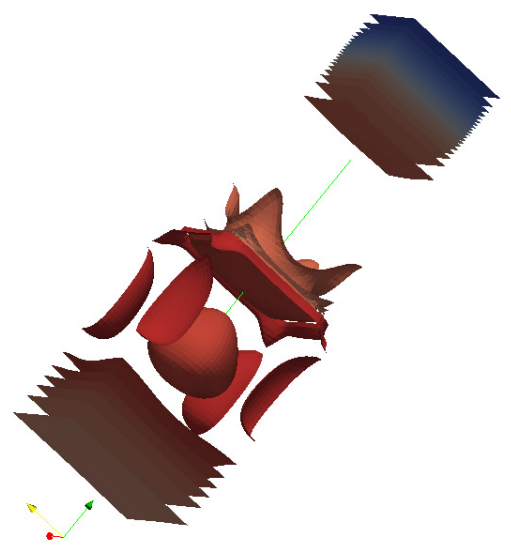

Figure 31. Les isosurfaces $d u$ maillage lâche

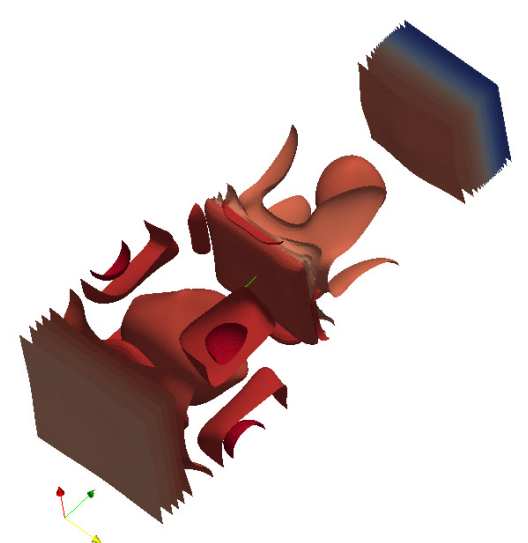

Figure 32. Les isosurfaces $d u$ maillage raffiné

de calcul en minutes pour des différents types de maillage du domaine entier et du quart du domaine. Ensuite, nous calculons dans la Figure 34 le rapport temporel défini par le quotient entre le coût de calcul 


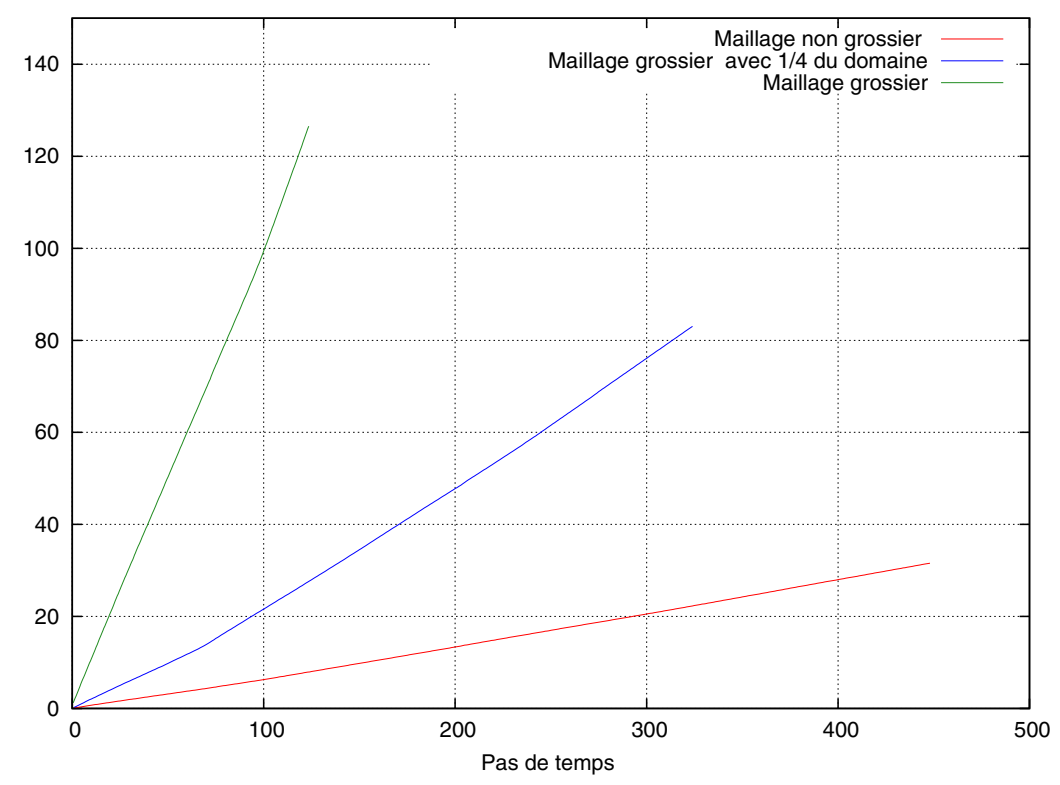

Figure 33. Coût de calcul en minutes

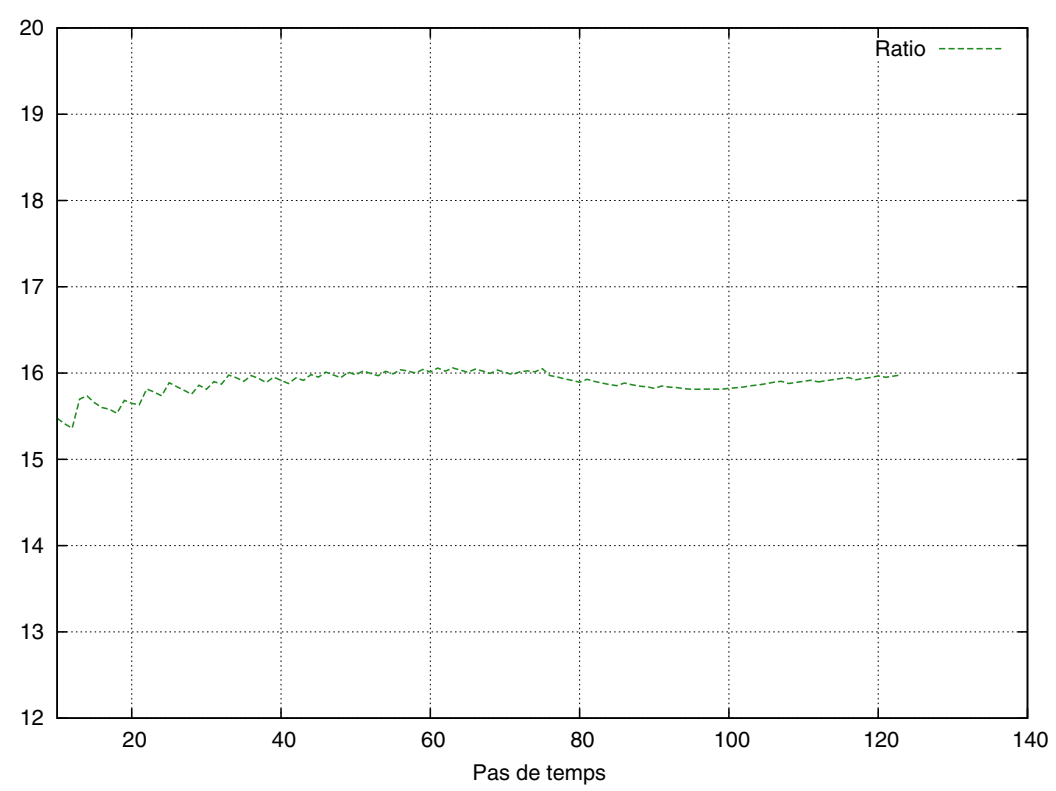

Figure 34. Ratio de coût de calcul

entre un maillage grossier et celui non grossier. Nous trouvons un ratio qui est proche de 16. Le ratio entre le temps de calcul associé au quart du maillage grossier et celui non grossier est relativement 4.

Nous réalisons un maillage cartésien lâche et raffiné sur le quart du domaine pour calculer le débit moyen calculé sur une section. Cette section est définie par la section orthogonale à la direction de l'écoulement. Ensuite, nous multiplions ce débit par 4 pour évaluer le débit moyen final. Ces deux maillages avec le pas de temps sont définis par:

$$
\left\{\begin{array}{l}
\text { Maillage lâche } \\
\Delta x=\Delta y=\Delta z=0.00125 m \\
n_{x}=12, n_{y}=12, n_{z}=80 \\
m_{x}=16, m_{y}=16, m_{z}=80 \\
\Delta t=1.128 \times 10^{-6}
\end{array}\right.
$$




$$
\left\{\begin{array}{l}
\text { Maillage raffiné } \\
\Delta x=\Delta y=\Delta z=0.000625 m \\
n_{x}=24, n_{y}=24, n_{z}=160 \\
m_{x}=32, m_{y}=32, m_{z}=160 \\
\Delta t=0.564 \times 10^{-6}
\end{array}\right.
$$

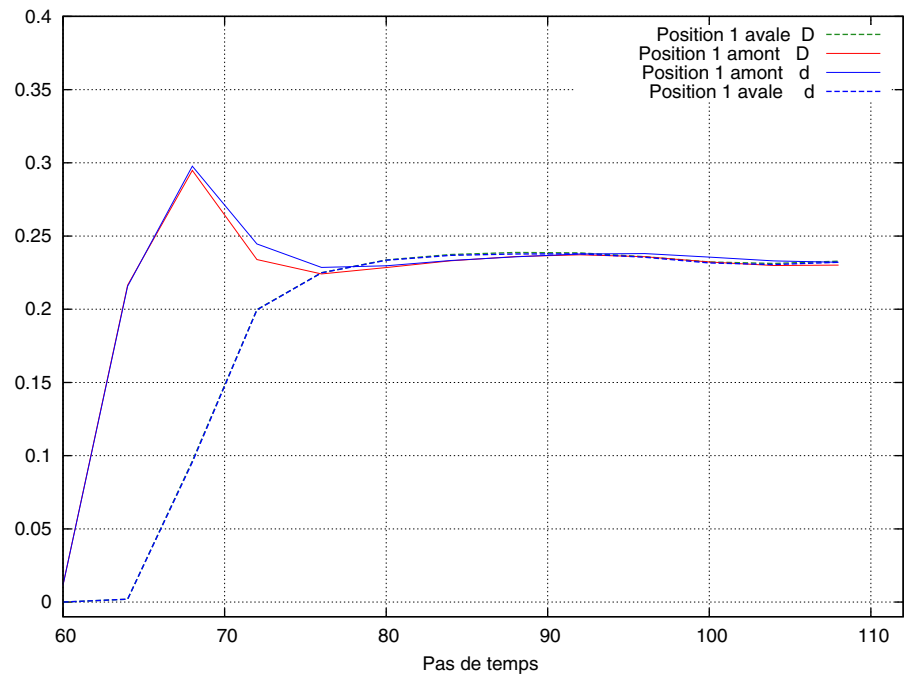

Figure 35. Débit moyen pour la position 1 pendant un aller associé à un maillage lâche du domaine cartésien

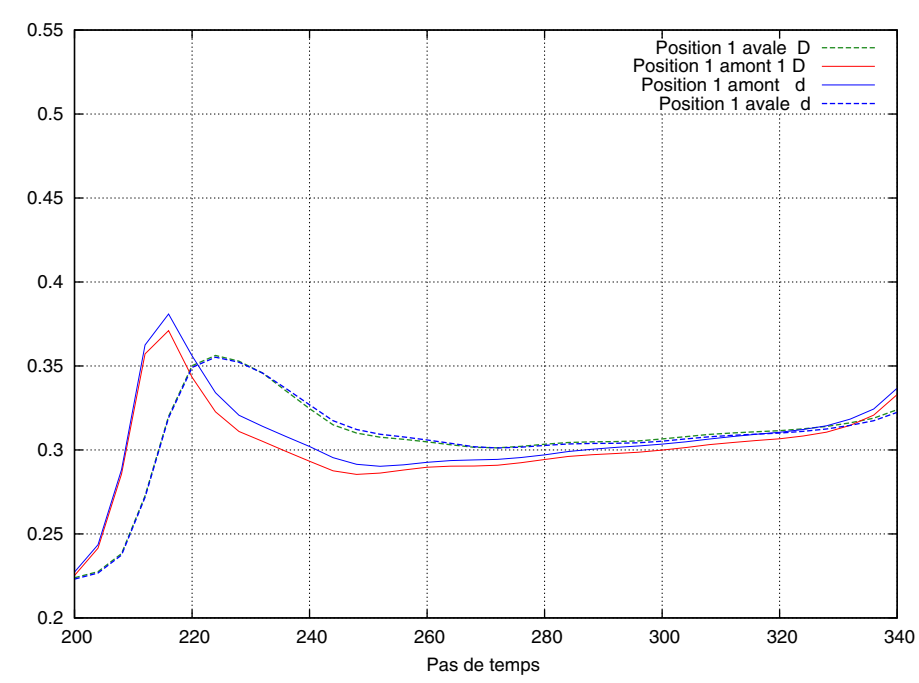

Figure 36. Débit moyen pour la position 1 pendant un retour associé à un maillage lâche du domaine cartésien

\subsubsection{La variation du débit moyen}

Nous avons montré avec les conditions I que notre maillage est bien convergent dans le sens où nous obtenons les mêmes grandeurs moyennes comme pour le débit moyen. À priori, nous ne pouvons pas prévoir avec les conditions initiales et aux limites à trouver un maillage convergent. C'est la raison pour laquelle, nous effectuons des simulations sur deux types différents de maillages. En comparaison avec les conditions I, nous considérons avec les conditions II qu'il y a une différence de température grande, et une vitesse d'éntrée très importante.

Nous allons maintenant décrire comment le débit moyen varie sur un domaine cartésien avec les conditions II pour l'onde d'aller et de retour. Nous remarquons que le débit moyen dépasse largement 0.3 dans la simulation définie dans la Figure 37 pour le maillage raffiné, et il ne dépasse pas 0.3 dans la simulation définie dans la Figure 35 pour un maillage lâche. Lorsque l'onde effectue son retour, il dépasse la valeur 0.4 concernant un maillage raffiné (voir Figure 38). En revanche, il ne la dépasse pas pour un maillage lâche (voir Figure 36). Par ailleurs, il y a un diphasage du débit moyen pendant une durée instationnaire courte. Ensuite, le régime permanent s'établit jusqu'à la fin de son retour.

Nous concluons que la structure du maillage avec les conditions II impact considérablement sur la simulation numérique pour le comportement du débit moyen. Contrairement aux conditions I où les deux types du maillage aboutissent aux mêmes résultats numériques. Par ailleurs, nous considérons que le domaine est cylindrique dans la simulation définie dans la Figure 39. Nous constatons pour un maillage 


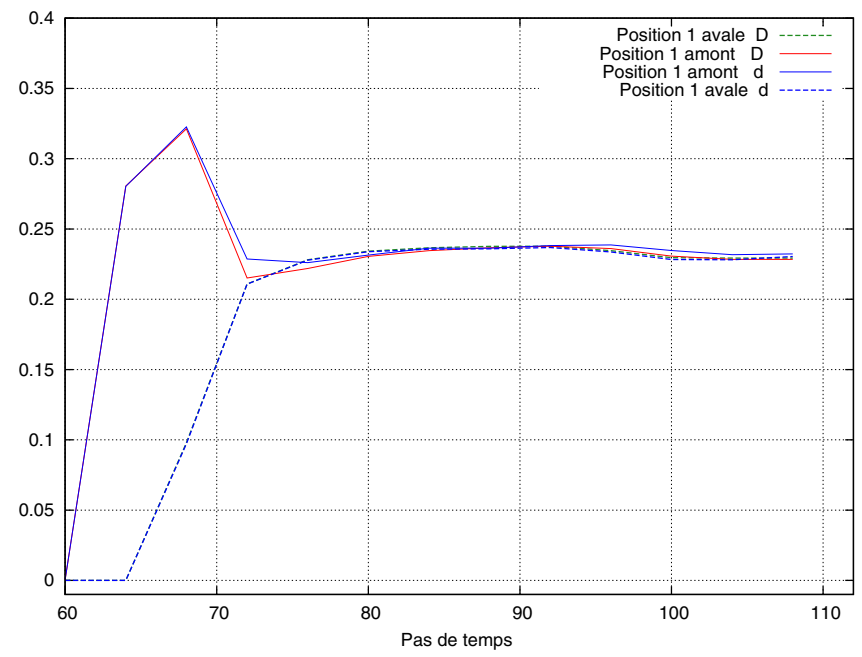

Figure 37. Débit moyen pour la position 1 pendant un aller associé à un maillage raffiné du domaine cartésien

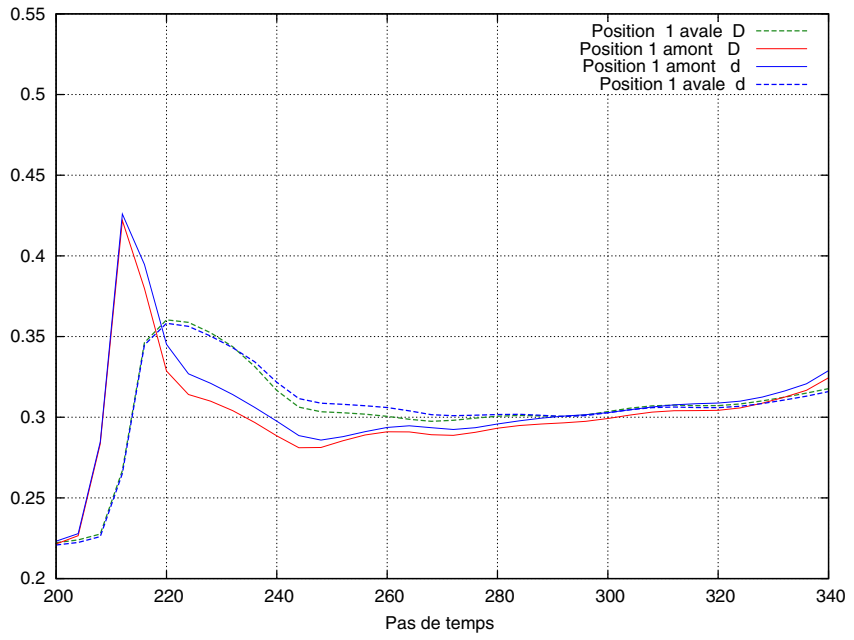

Figure 38. Débit moyen pour la position 1 pendant un retour associé à un maillage raffiné du domaine cartésien

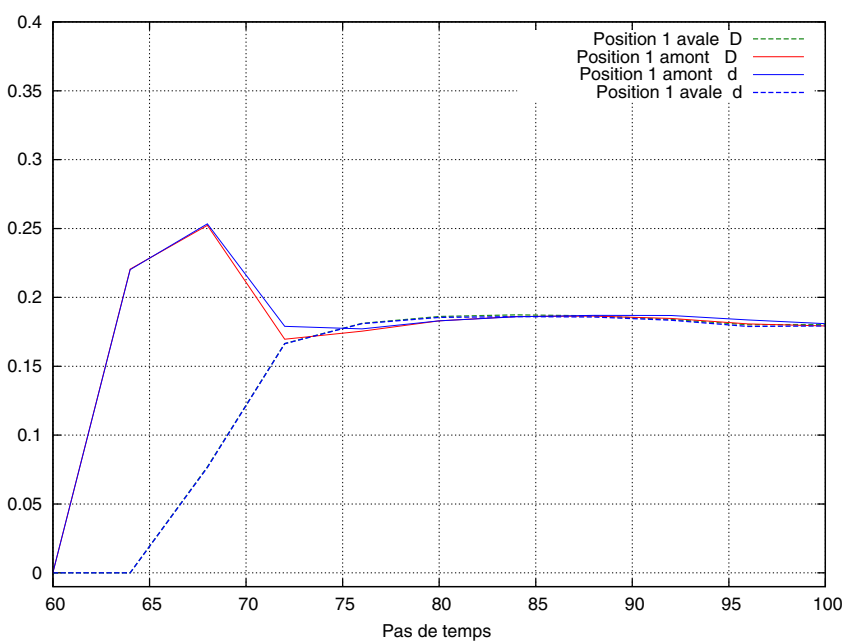

Figure 39. Débit moyen pour la position 1 pendant un aller associé à un maillage raffiné du domaine cylindrique

grossier que le débit moyen atteint la valeur maximale 0.25. Par ailleurs, le diphasage reste relativement le même.

\subsubsection{Le champs vectoriel et les isosurfaces}

Nous allons déterminer la nature de l'écoulement associé aux conditions II. Le champ vectoriel de vitesse induit un écoulement bidimensionnel proche de changement de section, et en particulier dans la deuxième partie de section. Concernant le maillage grossier, les vecteurs de vitesse se dirigent vers des directions aléatoires, plus précisément dans la deuxième partie gauche près du paroi. Le champs de vitesse contourne la partie près du paroi. Les vecteurs de vitesse prennent un sens régulier pour le maillage non raffiné.

Nous traçons des isosurafces pour ces deux types du maillages dans les Figures 42 et 43 . Pour le maillage non grossier, la simulation définie dans la Figure 42 montre que c'est un écoulement $1 \mathrm{D}$ en 
raison des plans parallèles. Cependant, pour le maillage raffiné, la simulation définie dans la Figure 43 caractérise un écoulement 3D puisque les plans ne sont pas parallèles.

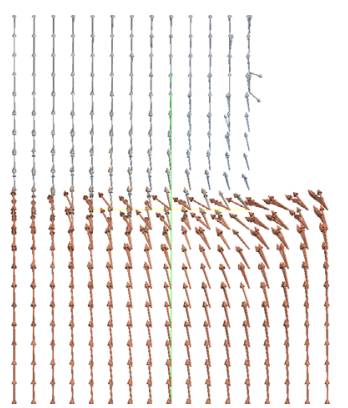

Figure 40. Champs de vecteurs pour un maillage non raffiné

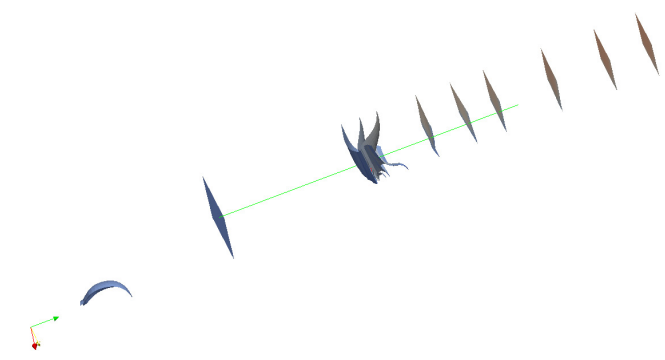

Figure 42. Les isosurfaces pour un maillage non raffiné

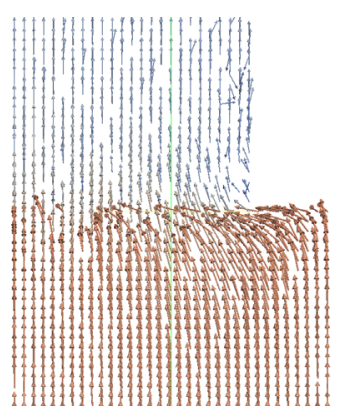

Figure 41. Champs de vecteurs pour un maillage raffiné

Figure 43. Les isosurfaces pour un maillage raffiné

\section{Conclusions et perspectives}

Nous avons montré à travers cet article la nécessité d'étudier des modèles de types d'orifices 3D en utilisant OpenFoam pour permettre de simuler l'écoulement de gaz dans des collecteurs d'échapement. L'objectif dans l'avenir est d'utiliser une approche de modélisation 0D/1D sur AMESim pour réaliser un couplage entre le modèle développé sur OpenFoam et celui-ci.

\section{Bibliographie}

OPENFOAM [online]. http: / /www . openfoam. com. Accessed : 2016-08-26.

MARIE-PIERRE DAINeSE. Simulation d'écoulements de fluide compressible en géométrie complexe : contribution à l'étude des schémas de discrétisation et d'algorithmes semi-implicites. PhD thesis, 1994.

GUO B., FLETCHER DF., and LANGRISH TAG., Effect of a downstream sudden contraction on flow instability behind a sudden pipe expansion. 2001.

Alexander Kurganov and Guergana Petrova. Central schemes and contact discontinuities. ESAIM : Mathematical Modelling and Numerical Analysis, 34(6):1259-1275, 2000.

AlEXANDER KURGANOV and EITAN TADMOR. New high-resolution central schemes for nonlinear conservation laws and convection-diffusion equations. Journal of Computational Physics, 160(1) :241-282, 2000.

HAIM NESSYAHU and EITAN TADMOR. Non-oscillatory central differencing for hyperbolic conservation laws. Journal of computational physics, 87(2) :408-463, 1990.

Satish G., Ashok Kumar K., Vara Prasad V., and Pasha Sk M., Comparison of flow analysis of a sudden and gradual change of pipe diameter using fluent software. Int. J. Res. Eng. Technol, 2(12) :41-45, 2013. 